\title{
Ambulatory Arrhythmia Monitoring in Pregnant Patients with Palpitations
}

\author{
Meredith O. Cruz, MD, MPH, MBA ${ }^{1}$ Judith U. Hibbard, MD ${ }^{1}$ Tamika Alexander, MD ${ }^{1}$ \\ Joan Briller, $\mathrm{MD}^{2}$ \\ ${ }^{1}$ Department of Obstetrics and Gynecology, Division of Maternal Fetal \\ Medicine, University of Illinois at Chicago, College of Medicine, \\ Chicago, Illinois \\ 2 Department of Medicine, Division of Cardiology, University of Illinois \\ at Chicago, College of Medicine, Chicago, Illinois \\ Address for correspondence and reprint requests Joan Briller, MD, \\ 840 South Wood Street, MC 715, Chicago, IL 60612 \\ (e-mail: briller@uic.edu). \\ Am J Perinatol 2013;30:53-58.
}

Palpitations, dizziness, and syncope are frequent complaints in pregnancy. In fact, Shotan et al documented that these complaints were among the most frequent cause of referral to the high-risk obstetric clinics at the University of Southern California medical center. ${ }^{1}$ In our institution, they account for approximately one-third of referrals to a cardiology clinic devoted to heart disease in pregnancy. An increased incidence of arrhythmias has been reported during pregnancy in patients with known heart disease, and adverse fetal outcomes occur more commonly in women who suffer antepartum arrhythmias compared with gravidas who do not have this disturbance. $^{2}$ The presence of unexplained palpitations, syncope, near syncope, or episodic dizziness is considered a class 1 indication for ambulatory electrocardiogram (ECG) monitoring according to recent guidelines published by the American College of Cardiology and the American Heart Association. ${ }^{3}$

Previous studies of pregnant women with arrhythmias have usually employed Holter testing, ECG with symptoms, or telemetry to assess for presence of arrhythmia.

Holter ambulatory monitors typically record the ECG continuously for a period of 24 to 48 hours and the patient documents symptoms occurring during that time frame. Unfortunately, the yield from Holter testing may be diminished compared with newer techniques as day-to-day variation in frequency of arrhythmias is high.

Ambulatory event loop recorders (ERTs) document the ECG in a continuous manner and store a few seconds in memory but only when an event marker is triggered by the received

December 14, 2011

accepted after revision

March 15, 2012

published online

July 26, 2012
Copyright $\odot 2013$ by Thieme Medical Publishers, Inc., 333 Seventh Avenue, New York, NY 10001, USA. Tel: +1(212) 584-4662.
DOI http://dx.doi.org/ 10.1055/s-0032-1321500. ISSN 0735-1631. 
patient at the time of symptoms. These devices can transfer data readily over conventional telephone lines and can be used for several weeks to identify infrequently occurring arrhythmias that might be missed with the use of conventional Holter monitoring. Newest event recorders have autocapture modes, which will automatically record abnormal rhythms meeting predetermined parameters even in the absence of symptoms. Event recorders were demonstrated by Kinlay et al to be twice as likely to provide a diagnostic rhythm strip during symptoms and to be more cost-effective when compared with Holter monitoring. ${ }^{4}$

In the current study, we assessed the frequency of various rhythm disturbances obtained following placement of a Holter monitor or an ERT in patients referred to the Women and Heart Disease program at the University of Illinois at Chicago (UIC) medical center for clinical symptoms. We planned a subgroup analysis of the ERT group. We hypothesized that benign arrhythmias would be a frequent finding and associated with benign outcomes in women referred for symptoms, but women with preexisting heart disease or arrhythmias would be more likely to have serious rhythm disturbances and poorer obstetric outcomes.

\section{Methods}

A retrospective chart review was performed for 96 gravidas who were referred to the UIC Heart Disease in Women Program for evaluation of unexplained palpitations, syncope, near syncope, or episodic dizziness who underwent clinically indicated ECG, Holter monitoring, or ERT from October 2002 through March 2008. Demographic data were obtained on the patients with regard to maternal age, race, gravidity, parity, body mass index (BMI), gestational age at referral and delivery, history of rhythm disturbance, heart disease, or structural heart disease (defined by echocardiography as showing congenital heart disease), left ventricular dysfunction, valvular heart disease, or coronary artery disease.

Additionally, functional class, 12-lead ECG abnormalities, electrolyte abnormalities (i.e., potassium, magnesium, and calcium levels), and history of hypertension, preeclampsia, diabetes, smoking, asthma, or presence of other disease that might affect the rhythm prevalence (e.g., thyroid disease, anemia) were recorded. Brain natriuretic peptide (BNP) levels and medications that might affect the presence of rhythm disturbances (e.g., $\beta$ blockers, digoxin) were documented. Histories of serious events such as congestive heart failure, transient ischemic attacks, cerebral vascular accidents, or maternal death were also recorded. Delivery information included mode of delivery, complications, birth weight, gestational age, and complications of prematurity such as respiratory distress syndrome, intraventricular hemorrhage, and perinatal demise.

ECGs, 24-hour Holter recordings, and ERTs were reviewed by the cardiologist, and arrhythmias were classified based upon a hierarchy according to severity of rhythm disturbance obtained. Both loop and autocapture recordings were analyzed. Rhythms identified were classified to the following groups: (1) sinus rhythm or sinus arrhythmia; (2) sinus or sinus arrhythmia with isolated premature atrial or ventricular contractions; (3) sinus tachycardia; (4) sinus tachycardia with isolated premature ventricular or atrial contractions; (5) supraventricular tachycardia; (6) atrial fibrillation or atrial flutter; (7) other (ventricular tachycardia, type 2 or greater heart block). Groups 1 to 4 were considered benign arrhythmias, and groups 5 to 7 were considered serious.

We performed a univariate analysis with chi-square calculations and Fischer exact tests, followed by a multivariate logistic regression analysis using the Hosmer and Lemeshow goodness-of-fit test. Factors with a $p$ value $<0.10$ in the univariate analysis were entered into the multivariate logistic regression. A $p$ value $<0.05$ was considered significant, and odds ratios (ORs), adjusted ORs and 95\% confidence intervals (CIs) were calculated.

\section{Results}

Demographic information for our patient population is illustrated in -Table 1 by overall group. Sixty-five gravidas

Table 1 Baseline Characteristics of the Patient Population

\begin{tabular}{|l|l|l|l|l|}
\hline Baseline Characteristics & Mean Event $(\boldsymbol{n}=\mathbf{6 5})$ & Mean Holter $(\boldsymbol{n}=\mathbf{1 9 )}$ & Mean ECG $(\boldsymbol{n}=\mathbf{1 2})$ & $\boldsymbol{p}$ Value \\
\hline Age $(\mathrm{y})$ & $26.1 \pm 6.6$ & $26.7 \pm 7.1$ & $30.5 \pm 7.3$ & 0.1 \\
\hline Primigravidas (\%) & 45.3 & 42.1 & 33.3 & 0.7 \\
\hline BMI & $29.7 \pm 7.4$ & $27.4 \pm 7.9$ & $28.7 \pm 6.8$ & 0.5 \\
\hline Gestational age at referral (wk $\pm \mathrm{d})$ & $22.6 \pm 8.0$ & $23.3 \pm 11.4$ & $23.4 \pm 10.5$ & 0.9 \\
\hline Gestational age at delivery (wk $\pm \mathrm{d})$ & $37.8 \pm 3.6$ & $39.0 \pm 1.3$ & $39.0 \pm 0.9$ & 0.3 \\
\hline Race (\%) & & & 75.0 & 0.4 \\
\hline African-American & 39.7 & 47.4 & 8.3 & \\
\hline Hispanic & 31.8 & 21.1 & 8.3 & \\
\hline Caucasian & 17.5 & 26.3 & 8.3 & \\
\hline Other & 11.1 & 5.3 & & \\
\hline
\end{tabular}

BMI, body mass index; ECG, electrocardiogram. 


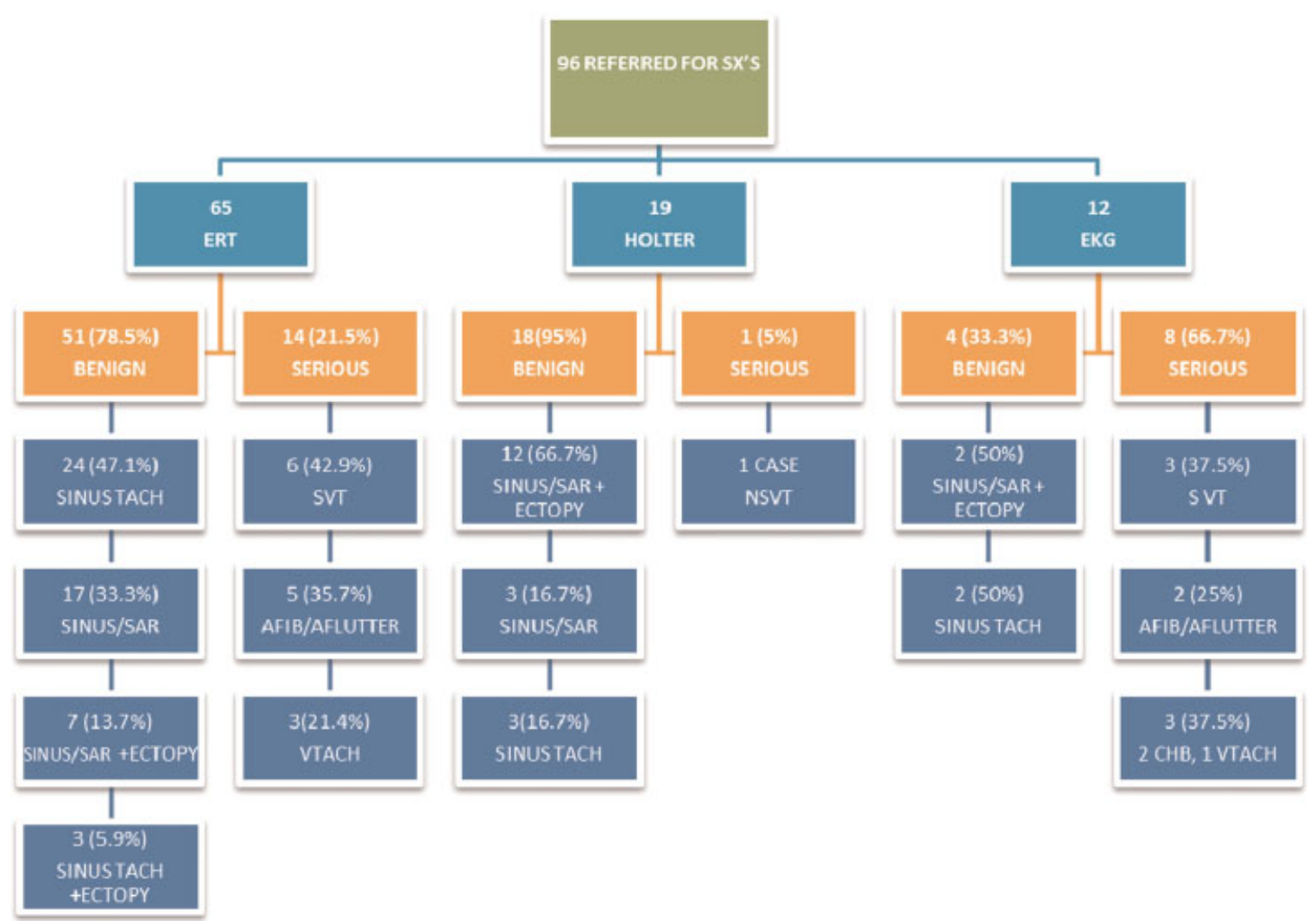

Figure 1 Composition of benign and serious arrhythmias that were found via all modalities. AFIB/AFLUTTER, atrial fibrillation or atrial flutter; CHB, complete heart block; NSVT, nonsustained ventricular tachycardia;SINUS/SAR, sinus rhythm or sinus arrhythmia; SINUS/SAR +ECTOPY, sinus rhythm or sinus arrhythmia with ectopy; SINUS TACH, sinus tachycardia; SINUS TACH +ECTOPY, sinus tachycardia with ectopy; SVT, supraventricular tachycardia; VTACH, ventricular tachycardia.

underwent ERT, 19 gravidas had Holter monitors, and for 12 gravidas diagnosis of rhythm disturbance responsible for symptoms was made by ECG. Of the 96 women, 73 (76\%) had benign arrhythmias and 23 (24\%) had serious arrhythmias. The benign and serious arrhythmias that were documented in our cohort are illustrated in - Fig. 1. Of interest, the mean gestational age at referral for symptoms was 22 weeks, the time in pregnancy when normal cardiovascular changes, such as cardiac output and blood volume, are reaching their maximum. Only four patients had left ventricular dysfunction at time of evaluation. An additional eight patients gave a history of congenital heart disease but only one had systemic ventricular dysfunction. No patients had significant pulmonary hypertension.

In the ERT group, 51 (78.5\%) had benign arrhythmias and 14 (21.5\%) had serious arrhythmias. Sinus tachycardia was the most common benign arrhythmia diagnosed, and supraventricular tachycardia was the most common serious arrhythmia diagnosed. Within the group of 65 women who underwent ERT, eight women had congenital heart disease, of whom five had valvular abnormalities; three women had a history of having cardiothoracic surgery; none had a history of coronary artery disease. Of these eight with congenital heart disease, only three were noted to have a serious rhythm disturbance.

In the Holter group, 18 (95\%) had benign arrhythmias and one patient (5\%) had a serious arrhythmia. Sinus/sinus ar- rhythmia with ectopy was the most common benign arrhythmia diagnosed, and there was one case of nonsustained ventricular tachycardia. In the ECG group, 4 (33.3\%) of these women had benign arrhythmias and 8 (66.7\%) had serious arrhythmias. Sinus/sinus arrhythmia with ectopy again was the most common benign arrhythmia diagnosed, and the serious arrhythmias were made up of three cases of supraventricular tachycardia, two cases of complete heart block, two cases of atrial fibrillation/atrial flutter, and one case of ventricular tachycardia.

To obtain more information on factors associated with serious arrhythmias in pregnancy, the three groups were combined and considered as a whole. - Table $\mathbf{2}$ presents the ORs and 95\% CIs for the variables analyzed. Variables analyzed included: history of cardiac disease or arrhythmia, electrolyte, hemoglobin, thyroid-stimulating hormone (TSH), BNP levels, history of diabetes, hypertension, asthma, or preeclampsia. We noted that women with a history of arrhythmias had an almost sixfold increase in experiencing an arrhythmia during gestation (OR 5.7, 95\% CI 2.1 to 15.5 , $p=0.0006$ ). Elevated calcium levels were noted to be protective for arrhythmias (OR $0.2,95 \% \mathrm{CI} 0.1$ to $0.9, p=0.03$ ) as were higher magnesium levels (OR 0.01, 95\% CI 0.001 to $0.4, p=0.02)$. There was a trend for obese women $\left(\mathrm{BMI} \geq 30 \mathrm{~kg} / \mathrm{m}^{2}\right.$ ) to experience a rhythm disturbance (OR $1.1,95 \%$ CI 1.0 to $1.1, p=0.05$ ). A diagnosis of serious rhythm 
Table 2 Univariate Analysis of Variables in Symptomatic Gravidas Possibly Associated with Arrhythmias

\begin{tabular}{|l|l|l|l|}
\hline Factor & OR & $95 \% \mathrm{Cl}$ & $p$ Value \\
\hline Structural heart disease & 2.0 & $0.7-5.5$ & 0.2 \\
\hline Congenital heart disease & 0.7 & $0.1-3.5$ & 0.7 \\
\hline History of CT surgery & 2.0 & $0.3-12.8$ & 0.5 \\
\hline Valvular heart disease & 0.5 & $0.1-3.9$ & 0.5 \\
\hline History of arrhythmia & 5.7 & $2.1-15.5$ & $0.0006^{\mathrm{a}}$ \\
\hline $\mathrm{K}(\mathrm{mmol} / \mathrm{L})$ & 0.7 & $0.2-2.8$ & 0.6 \\
\hline $\mathrm{Mg}(\mathrm{mg} / \mathrm{dL})$ & 0.01 & $<0.001-0.4$ & $0.02^{\mathrm{a}}$ \\
\hline $\mathrm{Ca}(\mathrm{mg} / \mathrm{dL})$ & 0.2 & $0.1-0.9$ & $0.03^{\mathrm{a}}$ \\
\hline $\mathrm{Hgb}(\mathrm{g} / \mathrm{dL})$ & 0.9 & $0.6-1.3$ & 0.5 \\
\hline TSH $(\mu \mathrm{lU} / \mathrm{mL})$ & 1.4 & $0.8-2.8$ & 0.3 \\
\hline BNP $(\mathrm{pg} / \mathrm{mL})$ & 2.9 & $0.7-12.1$ & 0.2 \\
\hline Diabetes & 0.8 & $0.2-4.1$ & 0.8 \\
\hline BMI $\left(\mathrm{kg} / \mathrm{m}^{2}\right)$ & 1.1 & $1.0-1.1$ & 0.05 \\
\hline Hypertension & 2.3 & $0.7-8.1$ & 0.2 \\
\hline Asthma & 1.6 & $0.4-5.9$ & 0.5 \\
\hline Preeclampsia & 2.7 & $0.4-21.2$ & 0.3 \\
\hline
\end{tabular}

BMI, body mass index; BNP, brain natriuretic peptide; $\mathrm{Cl}$, confidence interval; $\mathrm{CT}$, cardiothoracic; $\mathrm{Hgb}$, hemoglobin; OR, odds ratio; TSH, thyroid-stimulating hormone.

${ }^{a}$ Statistically significant.

disturbance in the entire population presenting with symptoms did not result in greater risk of cesarean section or induction of labor, nor having a newborn with cardiac arrhythmias prior to discharge from the hospital.

A multiple logistic regression analysis was then performed on the total group of patients presenting with symptoms. Because a history of a rhythm disturbance, structural heart defect, calcium levels, BNP levels, and hypertension were associated with arrhythmias from the univariate analysis at a significance level of at least $10 \%$, they were incorporated into the multivariate logistic regression analysis. Patients who had a history of previous arrhythmia were noted to have a much higher likelihood of recurrent arrhythmia during gestation (adjusted OR 8.5, 95\% CI 2.3 to 31.2, $p=0.01$. Again, we noted a trend for women with higher BMIs to experience an arrhyth- mia (adjusted OR 1.1, 95\% CI 1.0 to $1.6, p=0.1$ ). Similarly, elevated BNP level was associated with arrhythmia, but not significantly.

To examine further the factors associated with serious arrhythmias in pregnancy, and because ERT is now commonly our first-line diagnostic modality, a univariate analysis was performed on our subgroup that had the most subjects. As demonstrated in our univariate analysis in the ERT group $(-$ Table 3), we noted that obese women $(\mathrm{BMI} \geq 30)$ had a fourfold increased risk for the occurrence of serious rhythm disturbance during gestation (OR 4.0, 95\% CI 1.0 to 11.2 , $p=0.03$ ). Women with a history of arrhythmias had a fourfold increase in experiencing a serious rhythm disturbance during gestation (OR 4.7, 95\% $\mathrm{CI} 1.1$ to $20.3, p=0.01$ ). Other variables considered to be potentially associated with

Table 3 Univariate Analysis of Variables in the Event Recorder Group That May be Associated with Arrhythmias

\begin{tabular}{|l|l|l|l|}
\hline Factor & OR & $95 \% \mathrm{Cl}$ & $p$ Value \\
\hline History of LV dysfunction & 2.7 & $0.3-23.3$ & 0.3 \\
\hline History of arrhythmia & 4.7 & $1.1-20.3$ & $0.01^{\mathrm{a}}$ \\
\hline BMI $\geq 30$ & 4.0 & $1.0-11.2$ & $0.03^{\mathrm{a}}$ \\
\hline Delivery mode & 0.6 & $0.1-3.8$ & 0.07 \\
\hline Fetal arrhythmias p/birth & 4.9 & $0-85.0$ & 0.9 \\
\hline Fetal cardiac anomalies & 4.9 & $0-208.8$ & 0.3 \\
\hline
\end{tabular}

BMI, body mass index; $\mathrm{Cl}$, confidence interval; LV, left ventricular; OR, odds ratio.

${ }^{a}$ Statistically significant. 
arrhythmias, such as history of structural heart disease, ejection fraction, electrolytes (potassium, magnesium, calcium), hemoglobin, TSH, BNP, diabetes, asthma, hypertension, and preeclampsia, were not found to be significant and none were entered into the multiple regression analysis.

As a history of a serious rhythm and obesity were significantly associated with arrhythmias in the univariate analysis at a significance level of $10 \%$, they were incorporated into the multivariate logistic regression analysis. Patients with a history of previous serious rhythms were noted to be $20 \%$ more likely to have a recurrent serious arrhythmia during gestation when they presented with symptoms (adjusted $\mathrm{OR}=1.2,95 \% \mathrm{CI} 1.0$ to $1.5, p=0.08$ ), but this no longer reached statistical significance. However, the patients who had higher BMIs were more likely to have a serious arrhythmia (adjusted $\mathrm{OR}=1.1,95 \% \mathrm{CI} 1.0$ to $1.2, p=0.04$ ) in this model.

\section{Discussion}

As hypothesized, we found that benign arrhythmias were more frequently diagnosed than serious arrhythmias regardless of type of testing performed and that benign arrhythmias were associated with benign outcomes in women referred for symptoms. We also demonstrated that women with a history of arrhythmias had an eightfold increased risk of experiencing a serious rhythm disturbance during gestation, but not those with preexisting heart disease. However, contrary to our hypothesis, a diagnosis of serious rhythm disturbance did not result in greater chance of cesarean delivery, a greater risk for induction of labor, nor having a newborn with cardiac arrhythmias during the neonatal hospitalization. Our referral group was a relatively healthy group of women including only a small number of women with underlying structural heart disease, which likely explains our inability to demonstrate a relationship between structural heart disease and serious arrhythmia.

To our surprise, we found that obese women (BMI $\geq 30 \mathrm{~kg} /$ $\mathrm{m}^{2}$ ) in the ERT group had a fourfold increased risk in the occurrence of a serious rhythm disturbance during gestation. It may be that obese pregnant women have more sleep apnea and are predisposed to rhythm disturbances. We also discovered that higher calcium and magnesium levels were more protective against arrhythmias. Total calcium falls in pregnancy because of physiological hypoalbuminemia, but free calcium should not change. However, free calcium levels were not measured, and therefore the significance of this finding is unclear.

A higher percentage of malignant arrhythmias were detected with ERT ( $21 \%$ by ERT and $5 \%$ by Holter monitor) but due to the small numbers this was not significant. It would seem logical that a recorder worn for several weeks by a pregnant woman would be better able to detect an abnormality compared with a recorder worn for 24 to 48 hours, as Kinlay et al demonstrated in a nonpregnant population, although we cannot exclude a selection bias explaining the sensitivity of the two techniques as patients were sent for an event recorder or Holter monitor at the discretion of the ordering cardiologist. ${ }^{4}$ Moreover, a high percentage of women diagnosed by ECG had serious arrhythmias in comparison with other modalities. This also reflects a selection bias in that the women were symptomatic long enough for an ECG to be obtained during an arrhythmia and that once a diagnosis was made, no further evaluation was performed.

It also appears that BMI has a strong influence on the occurrence of serious rhythm disturbances detected during gestation by ERT. It is interesting that in the Women's Health Study, increased BMI was associated with both short- and long-term increase in atrial fibrillation risk. $^{5}$ Similarly, obesity-associated sleep disorder has been found to be associated with paroxysmal atrial fibrillation and nonsustained ventricular tachycardia. ${ }^{6}$ Obesity may contribute to arrhythmias through effects on heart rate as seen in ECG interval changes (increased QTc interval, QT dispersion, and repolarization abnormalities). ${ }^{7}$ Prolongation of the QT interval, a risk factor for arrhythmias, has been described with visceral obesity in healthy, premenopausal women. ${ }^{8}$ However, when we assessed the entire group of gravidas presenting with symptoms by multivariate analysis, BMI was no longer significant, which may be due to the fact that Holter testing has reduced sensitivity for arrhythmia detection in comparison to ERT. ERT should be more likely to detect a serious arrhythmia in all gravidas because she wears the monitor for a much longer period of time. ${ }^{4}$

Based on our findings, we believe that ERT is a better method of diagnosis in pregnant women, as it is in the nonpregnant population. Because women with a previous arrhythmia were more likely to be diagnosed with a serious arrhythmia, an aggressive diagnostic and management strategy is warranted. However, obstetric interventions may not be warranted in women with abnormal rhythm disturbances that have been adequately treated. Women with benign rhythm disturbances can be reassured that the risk of adverse outcome is low. Referral to cardiology should be performed in the setting of prior arrhythmias, obesity, and structural heart disease. An evaluation with a thorough history, physical exam, ECG, cardiac echo, and ERT is a reasonable initial diagnostic strategy. Our data may aid in streamlining diagnosis and therapy.

\section{Acknowledgment}

This study was supported by University of Illinois at Chicago (UIC) Center for Clinical and Translational Science (CCTS), Award Number UL1RR029879 from the National Center for Research Resources.

\section{References}

1 Shotan A, Ostrzega E, Mehra A, Johnson JV, Elkayam U. Incidence of arrhythmias in normal pregnancy and relation to palpitations, dizziness, and syncope. Am J Cardiol 1997;79:1061-1064

2 Silversides CK, Harris L, Haberer K, Sermer M, Colman JM, Siu SC. Recurrence rates of arrhythmias during pregnancy in women with 
previous tachyarrhythmia and impact on fetal and neonatal outcomes. Am J Cardiol 2006;97:1206-1212

3 Strickberger SA, Benson DW, Biaggioni I, et al; American Heart Association Councils on Clinical Cardiology, Cardiovascular Nursing, Cardiovascular Disease in the Young, and Stroke; Quality of Care and Outcomes Research Interdisciplinary Working Group; American College of Cardiology Foundation; Heart Rhythm Society. AHA/ACCF scientific statement on the evaluation of syncope: from the American Heart Association Councils on Clinical Cardiology, Cardiovascular Nursing, Cardiovascular Disease in the Young, and Stroke, and the Quality of Care and Outcomes Research Interdisciplinary Working Group; and the American College of Cardiology Foundation In Collaboration With the Heart Rhythm Society. J Am Coll Cardiol 2006;47: 473-484

4 Kinlay S, Leitch JW, Neil A, Chapman BL, Hardy DB, Fletcher PJ. Cardiac event recorders yield more diagnoses and are more costeffective than 48-hour Holter monitoring in patients with palpitations. A controlled clinical trial. Ann Intern Med 1996;124(1 Pt 1): $16-20$
5 Tedrow UB, Conen D, Ridker PM, et al. The long- and short-term impact of elevated body mass index on the risk of new atrial fibrillation the WHS (women's health study). J Am Coll Cardiol 2010;55:2319-2327

6 Monahan K, Storfer-Isser A, Mehra R, et al. Triggering of nocturnal arrhythmias by sleep-disordered breathing events. J Am Coll Cardiol 2009;54:1797-1804

7 Poirier P, Alpert MA, Fleisher LA, et al; American Heart Association Obesity Committee of Council on Nutrition, Physical Activity and Metabolism, Council on Cardiopulmonary Perioperative and Critical Care, Council on Cardiovascular Surgery and Anesthesia, Council on Cardiovas. Cardiovascular evaluation and management of severely obese patients undergoing surgery: a science advisory from the American Heart Association. Circulation 2009;120: 86-95

8 Peiris AN, Thakur RK, Sothmann MS, et al. Relationship of regional fat distribution and obesity to electrocardiographic parameters in healthy premenopausal women. South Med J 1991;84:961-965 